\title{
Analysis of factorial invariance across gender in the Taiwan version of the Satisfaction with Life Scale
}

\author{
Chia-huei $\mathrm{Wu}$, Grace Yao * \\ Department of Psychology, National Taiwan University, 1, Sec. 4, Roosevelt RD., Taipei, Taiwan
}

Received 4 April 2005; received in revised form 2 November 2005; accepted 3 November 2005

Available online 3 January 2006

\begin{abstract}
The purpose of this study was to examine factorial invariance of the 5-item Satisfaction with Life ScaleTaiwan version (SWLS) across gender among university students in Taiwan. A total of 476 undergraduate students (males $=207$ and females $=269$ ) at National Taiwan University completed the Taiwan version of the SWLS. Prior to any invariance analysis, one-factor and two-factor models (present and past factors) were applied to each group. The confirmatory factor analysis results revealed that both the one-factor model and the two-factor model were acceptable for each group. However, since the "present" and "past" factors were highly correlated in both groups $(>0.90)$, the one-factor model was accepted upon consideration of the principle of parsimony. Further, based on the one-factor model, results from multi-sample analysis revealed that the Taiwan version of the SWLS has the property of strict factorial invariance across gender, including invariance of factor loadings, unique variances and factor variance.
\end{abstract}

(c) 2005 Elsevier Ltd. All rights reserved.

Keywords: Factorial invariance; Satisfaction with Life Scale; Gender

\footnotetext{
${ }^{*}$ Corresponding author. Tel.: +886 2 33663098; fax: +8862 23629909.

E-mail address: kaiping@ntu.edu.tw (G. Yao).
} 


\section{Introduction}

As mentioned in many textbooks for general psychology, the ultimate goal for psychologists is to improve human beings' life quality by figuring out the process and mechanism of human mind and behaviors (e.g., Sternberg, 2001). Although there are various research divisions in psychology, however, the main purpose of each field is the same, that is, to improve humans' well-being. Subjective well-being (SWB) is an important research field that is highly relevant to the main purpose of psychology. In fact, many scholars have proposed their theories on SWB from different perspectives. For example, Ryff (1989) and Ryff and Keyes (1995) focused on the domains of SWB, especially for psychological well-being. They proposed that psychological well-being encompasses six distinct dimensions of wellness, including Autonomy, Environmental Mastery, Personal Growth, Positive Relations with Others, Purpose in Life, and Self-Acceptance. In addition, Ryan and Deci (2001) concentrated on the process of enhancing and detracting SWB. That is, they regarded SWB as the consequence of self-determination actions. They proposed that attainment of intrinsic life goals will provide relatively direct satisfaction of the basic needs, thus enhancing well-being, whereas the attainment of extrinsic life goals does not contribute to and may even detract from basic need satisfactions, leading to illbeing. Moreover, Heller, Watson, and Ilies (2004) discussed the sources of SWB by the framework of top-down (i.e., personological) and bottom-up (i.e., situational) forces on individuals' SWB.

Although these perspectives contributed to the progress of SWB research, they did not tap the basic issue on SWB - the nature of SWB. If we want to understand which domain SWB belongs to, which process can enhance SWB and which forces influence SWB, the first step is to identify the nature of SWB. In fact, Diener, Emmons, Larsen, and Griffin (1985), Lucas, Diener, and Suh (1996) have distinguished both affective and cognitive aspects of SWB. The affective aspect of SWB refers to the emotional component whereby levels of positive vs. negative affect were used to indicate the level of SWB. People who experienced more positive affect than negative affect were regarded as having higher SWB. Although this affective definition of SWB has been widely accepted and adopted in many empirical studies, SWB is also sometimes defined from a cognitive viewpoint, in which life satisfaction was used to describe the level of SWB (Diener et al., 1985; Pavot \& Diener, 1993). Life satisfaction refers to the conscious cognitive judgment of life in which individuals compare their life circumstances with a self-imposed standard (Diener et al., 1985). That is, individuals will report high life satisfaction if their perceived life circumstances are on a par with their own standard.

In order to measure an individual's SWB from the cognitive aspect, Diener et al. (1985) developed the Satisfaction with Life Scale (SWLS). Considering that different people may have very different ideas about what constitutes a good life, the SWLS was developed to assess satisfaction with one's life as a whole (Diener et al., 1985). The five global evaluation items are (a) In most ways my life is close to my ideal, (b) The conditions of my life are excellent, (c) I am satisfied with my life, (d) So far I have gotten the important things I want in life, and (e) If I could live my life over, I would change almost nothing.

The Satisfaction with Life Scale has been used extensively since 1985 and has good psychometric properties (Pavot \& Diener, 1993). Its internal consistency reliability coefficients ranged from 0.79 to 0.89 (Pavot \& Diener, 1993). The test-retest reliability coefficients of the SWLS 
were 0.83 for a 2-week interval and 0.84 for a 1-month interval (Pavot \& Diener, 1993). The SWLS also demonstrated adequate construct validity, convergent validity and discriminant validity (Arrindell, Heesink, \& Feij, 1999; Lucas et al., 1996; Pavot \& Diener, 1993; Sachs, 2003).

However, while many studies have demonstrated the good psychometric properties of the SWLS, some issues need to be addressed to further understand its psychometric properties and the nature of the cognitive aspect of SWB. The first issue is the factor structure of the SWLS. In the seminal work by Diener et al. (1985), the SWLS comprised only one factor and this single-factor solution has been replicated in different studies (Arrindell et al., 1999; Arrindell, Meeuwesen, \& Huyse, 1991; Atienza, Balaguer, \& Garcia-Merita, 2003; Lewis, Shevlin, Bunting, \& Joseph, 1995; Pavot, Diener, Colvin, \& Sandvik, 1991; Shevlin \& Bunting, 1994; Westaway, Maritz, \& Golele, 2003). Nevertheless, in Pavot and Diener's (1993) review paper, they indicated 'the item-total correlations and factor loadings suggest that the last item is the weakest in terms of convergence with other items. This may be because most of the items refer primarily to the present, whereas the fifth item refers primarily to the past, although this interpretation will require empirical testing (p. 167)'. Following this viewpoint, McDonald (1999) conducted confirmatory factor analysis (CFA) to test the two-factor model with "present" and "past" factors, in which the first three items were regarded as "present items" and the last two items were treated as "past items". McDonald (1999) reported that the two factors were highly correlated ( $r=0.86)$, hence, he proposed a hierarchical factor model to incorporate the "present" and "past" factors by a general factor of life satisfaction. Recently, Sachs (2003) performed similar work to test the factor structure of the SWLS (Hong Kong Chinese version). Sachs' (2003) result also revealed a larger correlation $(r=0.72)$ between the "present" and "past" factors in the two-factor model. However, inconsistent with McDonald's (1999) result, Sachs (2003) proposed a revised one-factor model after comparing several models. In the revised one-factor model, the five items were influenced by a single factor, but the uniqueness (error term) of the first two items was correlated. Sachs (2003) suggested that this finding may result from the redundant content of the two items. He believed that the items "In most ways my life is close to my ideal" and "The conditions of my life are excellent" may not have distinctive meanings among Hong Kong students. In addition, Sachs's (2003) results revealed that the two-factor model with "present" and "past" items was not a better framework to interpret the factor structure of the SWLS. Therefore, according to the existing literature, clarity is needed on whether "present" and "past" factors are meaningful for the SWLS.

Besides the factor structure of the SWLS, its factorial invariance needs to be addressed. Factorial invariance is an important property for a measurement that was developed to compare the mean level of a certain construct or trait among different groups, since interpretation of the mean differences may be problematic unless the underlying constructs are the same across groups. Therefore, when a measurement is used to compare mean differences among different groups, the measurement should have the same meaning across groups. Moreover, factorial invariance is an important property for case selection. Millsap and Kwok (2004) indicated that partial factorial invariance (when some, but not all, parameters are invariant) would debase the accuracy of selection when the composite score was used as the criterion for case selection. Thus, if a measurement is used to select cases with certain characteristics, the property of factorial invariance should be tenable if different groups are combined within a large sample. Past 
literature has discussed factorial invariance of the SWLS on gender and age. Shevlin, Brunsden, and Miles (1998) found that the SWLS has the property of factorial invariance across gender in a sample of British university students. However, in a sample of Spanish junior high school students, factorial invariance of the SWLS (Spanish version) was not obtained (Atienza et al., 2003). On factorial invariance across ages, Pons, Atienza, Balaguer, and Garcia-Merita (2000) analyzed the factorial invariance of the SWLS (Spanish version) across samples of adolescents (aged 11-15 yrs) and the elderly (aged 60-91 yrs). Results indicated that factor loadings and variances were not invariant between the two samples, suggesting the SWLS was sensitive to age.

Due to the inconsistent findings of previous studies, the issues of factor structure and factorial invariance of the SWLS need to be further addressed. On the issue of factor structure, the one-factor model and the two-factor model were compared to see if the framework of "present" and "past" factors was better than the one-factor model for Taiwanese people. This issue not only involved the psychometric aspect of the SWLS, but also related to the cultural aspect of SWB. Specifically, although different language versions of the SWLS keep the meaning of items consistently in translation, the factor analytic results cannot be generalized to populations from different cultural areas, because people from different cultures may have different definition, perception and interpretation of SWB (e.g., Diener \& Diener, 1995; Oishi, 2002; Oishi \& Diener, 2001, 2003; Suh, Diener, Oishi, \& Triandis, 1998). In addition, Lu's (2001) study did show that undergraduate students in Taiwan mentioned the time-oriented perspective on the nature of SWB. Some respondents mentioned that not only being satisfied with the present circumstance, but also holding a positive outlook for the future maintains an individual's SWB. In addition, some respondents mentioned that a past-and-present contrast would change their perception of SWB. These responses showed that the time reference of "past", "present" and "future" may influence the perception of SWB for Taiwanese people. Therefore, it is worth investigating if the two-factor model was better than the one-factor model in that culture.

Factorial invariance across gender was examined as the second issue. The gender issue was chosen since subgroups of males and females were not separated when further analysis was conducted in most empirical studies. This common practice implicitly assumes that items in the SWLS have the same meaning for male and female participants. However, only a few studies examine this property (e.g., Atienza et al., 2003; Shevlin et al., 1998). Therefore, examining the factorial invariance across gender is more important than that across ages, since elderly persons and young persons are not combined in practice. Accordingly, in this study, we have examined the factorial invariance across gender by the multi-sample analyses with CFA (Bollen, 1989).

\section{Method}

\subsection{Participants and procedure}

A total of 476 National Taiwan University undergraduate students participated in this study. Two hundred and seven of the participants were male (Mean age $=20.71, \mathrm{SD}=2.37$, 
aged between 18 and $30 \mathrm{yrs}$ ) and 269 of the participants were female (Mean age $=20.04$, $\mathrm{SD}=1.67$, aged between 18 and $30 \mathrm{yrs}$ ). All the participants completed the Taiwanese version of the Satisfaction with Life Scale in groups. Participants received a course credit for their participation.

\subsection{Instrument}

Satisfaction with Life Scale-Taiwan version. The Satisfaction with Life Scale (Diener et al., 1985) is a five-item scale, which employs a 7-point Likert scale with higher values corresponding to a higher degree of life satisfaction. The total score is calculated to represent a level of satisfaction, ranging from 5 to 35. Pavot and Diener (1993) proposed that a score of 20 represents the neutral point on the scale, the point at which the respondent is about equally satisfied and dissatisfied. For example, scores between 21 and 25 represent slightly satisfied, and scores between 15 and 19 represent slightly dissatisfied with life. Scores between 26 and 30 represent satisfied, and scores from 5 to 9 are indicative of being extremely dissatisfied with life. The SWLS Taiwan version was translated by the authors. Both forward and backward translations were conducted to make sure the concept of the SWLS Taiwan version was the same as the original version.

\subsection{Data analysis}

In factorial invariance analysis, a baseline model needs to be established prior to any invariance constraints. Thus, the first step is to determine whether a one- or a two-factor model should be adopted for each gender group. If the baseline model for each group is not the same, then the procedure of factorial invariance analysis should not be conducted. On the other hand, if the baseline model for each group is the same, and cannot be rejected in each group, restrictive constraints can then be imposed on the model. First, factor loadings were constrained to be equal across the gender groups to test for invariance of the factor loadings $\left(\Lambda_{\mathrm{m}}=\Lambda_{\mathrm{f}}\right)$. If the factor loading constrained model was acceptable, then unique variances of each item were constrained to be equal across males and females $\left(\Theta_{\mathrm{m}}=\Theta_{\mathrm{f}}\right)$. Finally, if factor loadings and unique variances of each item were equal across both groups, factor variance was constrained to be equal across gen$\operatorname{der}\left(\Phi_{\mathrm{m}}=\Phi_{\mathrm{f}}\right)$. For selection of both a one- or two-factor model, the analysis procedure was the same.

All the models were tested using covariance matrices (covariance matrices for each group are presented in the Appendix) and each model was estimated using the maximum likelihood method. The five items were not normally distributed. However, Muthén and Kaplan (1985) suggested that if variables have skewness and kurtosis from -1 to +1 , using likelihood estimators for non-normal categorical variables is acceptable. Since the skewness and kurtosis of the five items were between -1 and +1 , it was appropriate to use the maximum likelihood method for model estimation in this study. All the analyses were conducted using LISREL 8.0 (Joreskog \& Sorbom, 1993). Because the $\chi^{2}$ test is sensitive to sample size, information of other fit indices was used for model evaluation. 


\section{Results}

\subsection{Descriptive analysis of statistics}

Descriptive statistics for each item by gender are presented in Table 1, including the mean, standard deviation, skewness, and kurtosis. Item means and standard deviations of each gender group did not show significant differences (independent $t$ tests for two groups on all item means were not significant, all $p>.05$; Levene tests for two groups on all item variances were not significant, all $p>.05$ ). Also, skewness and kurtosis for each item were generally in the range of -1 to +1 for each group. Moreover, the present sample obtained a mean total score of 20.41 for males (neutral) and 21.10 for females (slightly satisfied). The two means were not significantly different $(t(474)=-1.33, p>.05)$.

\subsection{Multi-sample analysis}

Prior to any invariance analysis, a one-factor and a two-factor model were applied by gender. For the male group, both the one-factor model $\left(\chi^{2}(5)=16.51, p<.01\right.$; NFI $=.98$; NNFI $=.97$; $\mathrm{CFI}=.99 ; \mathrm{RMSEA}=.10 ; \mathrm{RMR}=.06)$ and the two-factor model $\left(\chi^{2}(4)=12.91, p<.05\right.$; $\mathrm{NFI}=.98 ; \mathrm{NNFI}=.97 ; \mathrm{CFI}=.99 ; \mathrm{RMSEA}=.10 ; \mathrm{RMR}=.05)$ were acceptable. According to the AIC and CAIC index, the values of each model were almost the same (AIC $=35.95$ for the one-factor model, and 33.77 for the two-factor model; CAIC $=79.28$ for the one-factor model, and 81.43 for the two-factor model). In addition, the "present" and "past" factors were highly correlated $(r=0.93)$. Thus, in considering the principle of parsimony, the one-factor model was retained for the male group.

For the female group, both the one-factor model $\left(\chi^{2}(5)=18.01, p<.01 ; \mathrm{NFI}=.98\right.$; $\mathrm{NNFI}=.97 ; \mathrm{CFI}=.99 ; \mathrm{RMSEA}=.10 ; \mathrm{RMR}=.06)$ and the two-factor model $\left(\chi^{2}(4)=13.09\right.$, $p<.05 ; \mathrm{NFI}=.99 ; \mathrm{NNFI}=.98 ; \mathrm{CFI}=.99 ; \mathrm{RMSEA}=.09 ; \mathrm{RMR}=.04)$ were acceptable According to the AIC and CAIC index, the values of each model were almost the same ( $\mathrm{AIC}=37.20$ for the one-factor model, and 34.63 for the two-factor model; CAIC $=83.15$ for the one-factor model, and 85.17 for the two-factor model). In addition, the "present" and "past" factors were highly correlated as well $(r=0.92)$. Thus, similarly, the one-factor model was retained for the female group.

Table 1

Descriptive statistics of each item for male and female groups

\begin{tabular}{|c|c|c|c|c|c|c|c|c|}
\hline \multirow[t]{2}{*}{ Sexes } & \multicolumn{4}{|c|}{ Male $(N=207)$} & \multicolumn{4}{|c|}{ Female $(N=269)$} \\
\hline & Mean & Std. & Skewness & Kurtosis & Mean & Std. & Skewness & Kurtosis \\
\hline Item1 & 3.97 & 1.32 & -.32 & -.50 & 4.08 & 1.22 & -.12 & -.43 \\
\hline Item2 & 4.04 & 1.31 & -.33 & -.38 & 4.24 & 1.26 & -.03 & -.39 \\
\hline Item 3 & 4.42 & 1.30 & -.41 & -.13 & 4.49 & 1.23 & -.25 & -.22 \\
\hline Item4 & 4.12 & 1.42 & -.19 & -.76 & 4.32 & 1.34 & -.14 & -.53 \\
\hline Item5 & 3.85 & 1.69 & -.02 & -1.06 & 3.97 & 1.63 & .10 & -.90 \\
\hline
\end{tabular}


Table 2

Parameter estimates of each model

\begin{tabular}{|c|c|c|c|c|c|c|c|c|c|c|c|c|c|c|}
\hline \multirow{3}{*}{$\begin{array}{l}\text { Model } \\
\text { Gender } \\
\text { Parameters }\end{array}$} & \multicolumn{4}{|c|}{ Unconstrained } & \multicolumn{4}{|c|}{$\Lambda_{\mathrm{m}}=\Lambda_{\mathrm{f}}$} & \multicolumn{4}{|c|}{$\Lambda_{\mathrm{m}}=\Lambda_{\mathrm{f}}, \Theta_{\mathrm{m}}=\Theta_{\mathrm{f}}$} & \multirow{2}{*}{\multicolumn{2}{|c|}{$\begin{array}{l}\text { Complete } \\
\text { invariance }\end{array}$}} \\
\hline & \multicolumn{2}{|l|}{ Male } & \multicolumn{2}{|l|}{ Female } & \multicolumn{2}{|l|}{ Male } & \multicolumn{2}{|l|}{ Female } & \multicolumn{2}{|l|}{ Male } & \multicolumn{2}{|l|}{ Female } & & \\
\hline & Unstd. & Std. & Unstd. & Std. & Unstd. & Std. & Unstd. & Std. & Unstd. & Std. & Unstd. & Std. & Unstd. & Std. \\
\hline$\lambda_{1}$ & 1.00 & 0.83 & 1.00 & 0.86 & 1.00 & 0.84 & 1.00 & 0.88 & 1.00 & 0.86 & 1.00 & 0.85 & 1.00 & 0.86 \\
\hline$\lambda_{2}$ & 0.99 & 0.82 & 0.94 & 0.84 & 0.96 & 0.83 & 0.96 & 0.81 & 0.96 & 0.83 & 0.96 & 0.81 & 0.96 & 0.82 \\
\hline$\lambda_{3}$ & 1.03 & 0.87 & 0.98 & 0.89 & 1.00 & 0.87 & 1.00 & 0.87 & 1.01 & 0.87 & 1.01 & 0.86 & 1.01 & 0.86 \\
\hline$\lambda_{4}$ & 1.00 & 0.77 & 0.89 & 0.79 & 0.94 & 0.75 & 0.94 & 0.73 & 0.94 & 0.76 & 0.94 & 0.74 & 0.94 & 0.74 \\
\hline$\lambda_{5}$ & 0.98 & 0.63 & 0.95 & 0.64 & 0.96 & 0.64 & 0.96 & 0.63 & 0.97 & 0.65 & 0.97 & 0.62 & 0.97 & 0.63 \\
\hline$\delta_{1}$ & 0.55 & 0.32 & 0.32 & 0.35 & 0.54 & 0.30 & 0.33 & 0.23 & 0.43 & 0.25 & 0.43 & 0.28 & 0.43 & 0.27 \\
\hline$\delta_{2}$ & 0.53 & 0.31 & 0.56 & 0.32 & 0.54 & 0.31 & 0.56 & 0.35 & 0.55 & 0.32 & 0.55 & 0.35 & 0.55 & 0.33 \\
\hline$\delta_{3}$ & 0.42 & 0.25 & 0.39 & 0.26 & 0.42 & 0.25 & 0.38 & 0.25 & 0.40 & 0.24 & 0.40 & 0.26 & 0.40 & 0.25 \\
\hline$\delta_{4}$ & 0.82 & 0.41 & 0.87 & 0.43 & 0.84 & 0.43 & 0.86 & 0.46 & 0.84 & 0.43 & 0.84 & 0.46 & 0.84 & 0.45 \\
\hline$\delta_{5}$ & 1.73 & 0.60 & 1.59 & 0.63 & 1.72 & 0.60 & 1.59 & 0.60 & 1.65 & 0.58 & 1.65 & 0.61 & 1.65 & 0.60 \\
\hline$\psi_{11}$ & 1.19 & 1.00 & 1.17 & 1.00 & 1.26 & 1.00 & 1.13 & 1.00 & 1.26 & 1.00 & 1.11 & 1.00 & 1.18 & 1.00 \\
\hline
\end{tabular}

Unstd. $=$ Unstandardized estimates; Std. $=$ standardized estimates.

Since the two baseline models for each gender were the same, multi-sample analysis was then conducted. First, a multi-sample analysis with the unconstrained model showed an acceptable baseline model for both males and females $\left(\chi^{2}(10)=34.52, p<.05\right.$; NFI $=.98 ; \mathrm{NNFI}=.97$; $\mathrm{CFI}=.99$; RMSEA $=.10$ ). The parameter estimates of this model are presented in the second column of Table 2. Then, to test the invariance of the factor loadings across gender, factor loadings were constrained to be equal across the two groups. Multi-sample analysis revealed that this constrained model was acceptable $\left(\chi^{2}(14)=35.77, p<.05\right.$; NFI $=.98$; NNFI $=.98$; CFI $=.99$; RMSEA $=.08)$. Also, the $\chi^{2}$ difference test between baseline model and constrained model was not significant $\left(\chi^{2}(4)=1.25, p>0.05\right)$, suggesting that factor loadings of both gender groups were invariant. The parameter estimates of this model are presented in the third column of Table 2.

In addition to the factor loadings, unique variances of each item were also constrained to be equal across the two groups. Multi-sample analysis showed that this constrained model was acceptable $\left(\chi^{2}(19)=44.74, p<.05 ; \mathrm{NFI}=.98 ; \mathrm{NNFI}=.98 ; \mathrm{CFI}=.99\right.$; $\left.\mathrm{RMSEA}=.07\right)$. Moreover, the $\chi^{2}$ difference test between the two constrained models was not significant $\left(\chi^{2}(5)=8.97, p>0.05\right)$. This suggested that, aside from the factor loadings, unique variances of each item were also invariant across gender. The parameter estimates of this model are presented in the fourth column of Table 2.

Finally, besides the constraints mentioned above, factor variances were also constrained to be equal across the two groups. Multi-sample analysis revealed this constrained model was acceptable $\left(\chi^{2}(20)=45.47, p<.01 ; \mathrm{NFI}=.97\right.$; NNFI $\left.=.99 ; \mathrm{CFI}=.99 ; \mathrm{RMSEA}=.07\right)$. Additionally, the $\chi^{2}$ difference test between the two constrained models was not significant $\left(\chi^{2}(1)=0.73\right.$, $p>0.05)$. Therefore, all these results revealed that the factor loadings, unique variances and factor variances were invariant across gender. The parameter estimates of this complete invariance model are presented in the fifth column of Table 2. 


\section{Discussion}

The present study supported the single-factor structure of the SWLS Taiwan version, and revealed the SWLS Taiwan version was factor invariant across gender in a sample of Taiwan university students. Consistent with McDonald (1999) and Sachs (2003), "present" and "past" factors in the two-factor model were highly correlated for each group, suggesting that the "present" and "past" factors could not be differentiated among these groups and one factor is enough to explain the SWLS structure among undergraduate students in Taiwan. However, this finding was not consistent with Lu's (2001) study, in which undergraduate students mentioned a time-oriented perspective of SWB. We think this inconsistent finding may result from the different task in the current study and Lu's (2001) study. In her study, students were asked to provide a personal definition of SWB. But in this study, students were asked to rate their SWB on the SWLS. We think that although students have a time-oriented perspective of SWB, it may not have a great impact for them in SWB evaluation, because students' life experiences are relatively stable. Thus, the past and present SWB did not represent dramatically different meanings of SWB. However, we did not exclude the possibility that the two-factor model with "present" and "past" factors could be a better factor structure of the SWLS when certain populations are assessed. Zimbardo and Boyd (1999), Boniwell and Zimbardo (2004), Boniwell (2005) and Pavot et al. (1998) indicated that a "temporal dimension" or "time perspective" is an important factor for understanding many aspects of human behavior and individuals' SWB. Boniwell's (2005) study revealed that "time perspective" and "perceived use of time" have significant relations with various SWB measures. Moreover, Pavot, Diener, and Suh (1998) even developed a temporal Satisfaction with Life Scale to capture the temporal meaning of the SWB. In their study, the factor analysis result did support the three-factor structure of the temporal model on SWB, which contains the past, present and future factors of SWB. Therefore, based on these studies, it is worth examining the two-factor model of the SWLS in the future with certain populations that might be sensitive to "present" and "past" life events, such as elderly, sick groups or people who have experiences of dramatic changes of life, to show the temporal effect of SWB.

Further, regarding the issue of factorial invariance, this study revealed that the SWLS Taiwan version has the property of factorial invariance across gender, indicating that the strength of the relationships between each item and the underlying construct is the same for males and females. This finding was consistent with Shevlin et al. (1998). However, for other age populations, factorial invariance across gender of the SWLS might not be defensible. For example, the results from Atienza et al.'s (2003) study showed that factor loadings and unique variances were not invariant across gender in a sample of Spanish junior high school students. Thus, according to the results from the current study, Shevlin et al.'s (1998) study and Atienza et al.'s (2003) study, it suggests that age may be related to gender factorial invariance of the SWLS. However, since these three studies were conducted in different cultures, this suggestion is speculative. In the future, cross-gender factorial invariance of the SWLS can be investigated in different age groups, such as senior high school students, junior high school students, adults, and seniors within a culture to ascertain if the items of the SWLS have different meanings to male and female respondents in different age populations. 


\section{Appendix A}

Item covariance matrices of each gender group

\begin{tabular}{lccccc}
\hline & Item1 & Item2 & Item3 & Item4 & Item5 \\
\hline Male $(N=207)$ & & & & & \\
Item1 & 1.747 & 1.225 & 1.187 & 1.183 & 1.253 \\
Item2 & 1.225 & 1.712 & 1.258 & 1.136 & 0.992 \\
Item3 & 1.187 & 1.258 & 1.691 & 1.250 & 1.199 \\
Item4 & 1.183 & 1.136 & 1.250 & 2.019 & 1.305 \\
Item5 & 1.253 & 0.992 & 1.199 & 1.305 & 2.866 \\
Female & $N=269)$ & & & & \\
Item1 & 1.493 & 1.126 & 1.139 & 1.000 & 1.171 \\
Item2 & 1.126 & 1.594 & 1.097 & 0.957 & 0.896 \\
Item3 & 1.139 & 1.097 & 1.520 & 1.056 & 1.076 \\
Item4 & 1.000 & 0.957 & 1.056 & 1.792 & 1.137 \\
Item5 & 1.171 & 0.896 & 1.076 & 1.137 & 2.652 \\
\hline
\end{tabular}

\section{References}

Arrindell, W. A., Heesink, J., \& Feij, J. A. (1999). The Satisfaction with Life Scale (SWLS): Appraisal with 1700 healthy young adults in The Netherlands. Personality and Individual Differences, 26, 815-826.

Arrindell, W. A., Meeuwesen, L., \& Huyse, F. J. (1991). The Satisfaction with Life Scale (SWLS): Psychometric properties in a non-psychiatric medical outpatients sample. Personality and Individual Differences, 12, 117-123.

Atienza, F. L., Balaguer, I., \& Garcia-Merita, M. L. (2003). Satisfaction with Life Scale: Analysis of factorial invariance across sexes. Personality and Individual Differences, 35, 1255-1260.

Bollen, K. A. (1989). Structural equations with latent variables. New York: John Wiley.

Boniwell, I. (2005). Beyond time management: How the latest research on time perspective and perceived time use can assist clients with time-related concerns. International Journal of Evidence Based Coaching and Mentoring, 3, 61-74.

Boniwell, I., \& Zimbardo, P. G. (2004). Balancing time perspective in pursuit of optimal functioning. In P. A. Linley \& S. Joseph (Eds.), Positive psychology in practice. New Jersey: John Wiley \& Sons.

Diener, E., \& Diener, M. (1995). Cross-cultural correlates of life satisfaction and self-esteem. Journal of Personality and Social Psychology, 68, 653-663.

Diener, E., Emmons, R. A., Larsen, R. J., \& Griffin, S. (1985). The Satisfaction with Life Scale. Journal of Personality Assessment, 49, 71-75.

Heller, D., Watson, D., \& Ilies, R. (2004). The role of person vs. situation in life satisfaction: A critical examination. Psychological Bulletin, 130, 574-600.

Joreskog, K. G., \& Sorbom, D. (1993). LISREL 8: Structural equation modeling with the SIMPLIS command language. Hillsdale, NJ: Lawrence Erlbaum Associates.

Lewis, C. A., Shevlin, M. E., Bunting, B. P., \& Joseph, S. (1995). Confirmatory factor analysis of the Satisfaction with Life Scale: Replication and methodological refinement. Perceptual and Motor Skills, 80, 304-306.

Lu, L. (2001). Understanding happiness: A look into the Chinese folk psychology. Journal of Happiness Studies, 2, 407-432.

Lucas, R. E., Diener, E., \& Suh, E. (1996). Discriminant validity of well-being measures. Journal of Personality and Social Psychology, 71, 616-628.

McDonald, R. P. (1999). Test theory: A unified treatment. Mahwah, NJ: Lawrence Erlbaum. 
Millsap, R. E., \& Kwok, O. M. (2004). Evaluating the impact of partial factorial invariance on selection in two populations. Psychological Methods, 9, 93-115.

Muthén, B., \& Kaplan, D. (1985). A comparison of some methodologies for the factor analysis of non-normal Likert variables. British Journal of Mathematical and Statistical Psychology, 38, 171-189.

Oishi, S. (2002). The experiencing and remembering of well-being: A cross-cultural analysis. Personality and Social Psychology Bulletin, 28, 1398-1406.

Oishi, S., \& Diener, E. (2001). Goals, culture, and subjective well-being. Personality and Social Psychology Bulletin, 27, $1674-1682$.

Oishi, S., \& Diener, E. (2003). Culture and well-being: The cycle of action, evaluation and decision. Personality and Social Psychology Bulletin, 29, 939-949.

Pavot, W., \& Diener, E. (1993). Review of the Satisfaction with Life Scale. Psychological Assessment, 5, 164-172.

Pavot, W., Diener, E., Colvin, C. R., \& Sandvik, E. (1991). Further validation of the Satisfaction evidence for the crossmethod convergence of well-being. Social Indicators Research, 28, 1-20.

Pavot, W., Diener, E., \& Suh, E. (1998). The Temporal Satisfaction with Life Scale. Journal of Personality Assessment, 70, 340-354.

Pons, D., Atienza, F. L., Balaguer, I., \& Garcia-Merita, M. L. (2000). Satisfaction with Life Scale: Analysis of factorial invariance for adolescents and elderly persons. Perceptual and Motor Skills, 91, 62-68.

Ryan, R. M., \& Deci, E. L. (2001). Self-determination theory and the facilitation of intrinsic motivation, social development, and well-being. American Psychologist, 55, 68-78.

Ryff, C. D. (1989). Happiness is everything, or is it. Explorations on the meaning of psychological well-being. Journal of Personality and Social Psychology, 57, 1069-1081.

Ryff, C. D., \& Keyes, C. L. M. (1995). The structure of psychological well-being revisited. Journal of Personal and Social Psychology, 69, 719-727.

Sachs, J. (2003). Validation of the Satisfaction with Life Scale in a sample of Hong Kong University students. Psychologia, 46, 225-234.

Shevlin, M. E., Brunsden, V., \& Miles, J. N. V. (1998). Satisfaction with Life Scale: Analysis of factorial invariance, mean structures and reliability. Personality and Individual Differences, 25, 911-916.

Shevlin, M. E., \& Bunting, B. P. (1994). Confirmatory factor analysis of the Satisfaction with Life Scale. Perceptual and Motor Skills, 79, 1316-1318.

Sternberg, R. J. (2001). Psychology: In search of the human mind. Fort Worth, TX: Harcourt College Publishers.

Suh, E., Diener, E., Oishi, S., \& Triandis, H. C. (1998). The shifting basis of life satisfaction judgments across cultures: Emotions versus norms. Journal of Personality and Social Psychology, 74, 482-493.

Westaway, M. S., Maritz, C., \& Golele, N. J. (2003). Empirical testing of the Satisfaction with Life Scale: A South African pilot study. Psychological Reports, 92, 551-554.

Zimbardo, P. G., \& Boyd, J. N. (1999). Putting time in perspective: A valid reliable individual-differences metric. Journal of Personality and Social Psychology, 77, 1271-1288. 